\title{
Editorial: special issue "NANO-2018"
}

\section{Olena Fesenko ${ }^{1}$}

Published online: 23 July 2020

(c) King Abdulaziz City for Science and Technology 2020

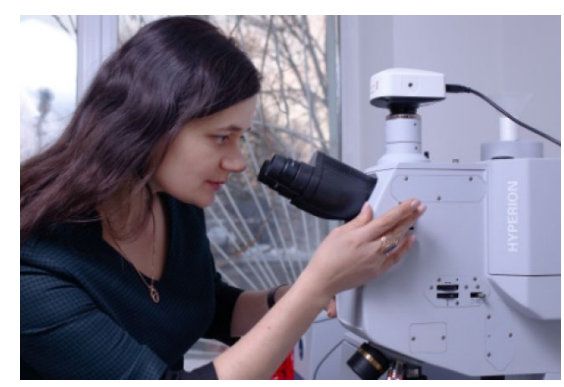

This special issue contains the manuscripts of contributions presented at the international research and practice conference "Nanotechnology and Nanomaterials" (NANO2018) that was held in Kiev from August 27 to 30, 2018. The conference focused on the latest advances in nanosciences and nanotechnologies and promoted profound scientific discussions between scientists and researchers from various fields from classic physics to biotechnology. Nanotechnology is becoming an important field of diagnostics in physics, chemistry, and medicine. This special issue disseminates interdisciplinary knowledge and scientific news on cuttingedge research in various nano-related research fields.

NANO-2018 allowed the participants, including young scientists, to familiarize themselves with current research and application problems in this area and thus to promote further implementation of nanotechnologies into innovations in accordance to public needs. From year to year, the annual international NANO conference grows and attracts more attention and participants. In 2018 the Organizing Committee of the NANO-2018 conference had received more than 800 application forms from about 30 countries across the world. The conference also gave the opportunity to young

Olena Fesenko

fesenko@iop.kiev.ua

1 International Science and Innovation Department, Surface Enhanced Spectroscopy (SES) Laboratory, Institute of Physics of the National Academy of Science of Ukraine, Kiev, Ukraine and early-career researchers to attend lectures by internationally recognized experts and roundtable discussions on the emerging fields in nanosciences and nanotechnologies.

The NANO-2018 conference brought together leading scientists and young researchers from many countries of the world. The conference topics were as follows: nanobiotechnology for healthcare; nanochemistry and biotechnology; nanocomposites and nanomaterials; nanoobject microscopy; nanooptics and photonics; nanoplasmonics and surface enhanced spectroscopy; nanoscale physics; nanostructured surfaces; physico-chemical nanomaterials science.

This special issue on the NANO-2018 conference contains top research articles covering all aspects of this multidisciplinary science, including biotechnology, electronics and photonics, quantum phenomena, sensing, patterning and fabrication, nanomaterial synthesis and analysis of their properties. Here you can find new knowledge which includes, without being limited to, the following themes: biogenic approach and antibacterial activity of copper oxide nanoparticles; highly sensitive graphene quantum dot sensors for acetone detection in breath from individuals with diabetes mellitus; about optical and electrical properties of different types of metal complex nanocomposites; new methods of biosynthesis of highly stable fluorescent metal nanoparticles; new antioxidant, anticancer, and nano-toxicological properties of silver nanoparticles; toxic effects of different metal nanoparticles and their influence on mesenchymal stem cells; 3D printed polyvinyl alcohol tablets; nanomechanical and nanotribological characterization of self-lubrication for bearing applications; optical and electrical properties of nanowires for solar cells; enhanced electrochemical efficiency in vanadium redox flow battery, etc.

Also, this special issue "NANO-2018" presents different types of nanobiotechnology applications at the frontiers of research in the hybrid discipline of nanoscience and biotechnology with equal contributions, such as using different metal and semiconductor nanoparticles for medicine; using graphene-like materials for nanoelectronics and nanooptics; using different types of nanostructures for nanoplasmonics, etc. 
This special issue "NANO-2018" is intended for all stakeholders in nanoscience and nanotechnology research from $\mathrm{PhD}$ students, researchers, and technicians to industrial engineers.

Dr. Olena Fesenko (Guest Editor) 